\title{
SISTEMAS DE MANEJO EN CAFÉ (Coffea arabica L.) Y SU EFECTO EN LA FERTILIDAD DEL SUELO Y EL
} RENDIMIENTO DEL CAFÉ ORO, MASATEPE, MASAYA

\section{CROPPING SYSTEMS IN COFFEE (Coffea arabica L.), AND ITS EFFECT ON SOIL FERTILITY AND YIELD OF GREEN COFFEE BEANS, MASATEPE, MASAYA}

\author{
Luna-Rodríguez Fátima del Carmen ${ }^{1}$, López-Mena Jaquelinne Guissel ${ }^{1}$, Larios-González Roberto ${ }^{2}$ \\ ${ }^{1}$ Graduadas de la Universidad Nacional Agraria, Facultad de Agronomía \\ ${ }^{2}$ Docente Universidad Nacional Agraria, Facultad de Agronomía. Dirección de Investigación, Extensión y Posgrado
}

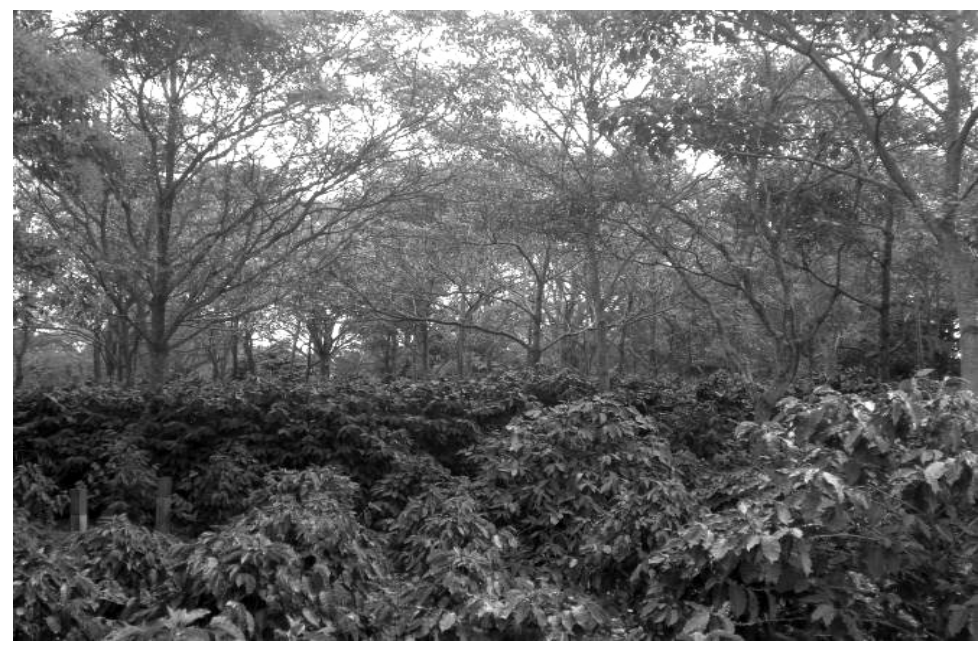

\section{RESUMEN}

El objetivo de este estudio fue comparar la influencia de tres sistemas de manejo en café sobre la fertilidad física, química y biológica del suelo y el rendimiento en café oro en el municipio de Masatepe, departamento de Masaya, Nicaragua en el período de julio del 2009 a enero del 2010. El tratamiento orgánico intensivo (OI) estuvo conformado por dos especies leguminosas como sombra, Inga laurina (Sw.) Willd (guaba) y Samanea saman (Jacq.) Merrill (genízaro), más aplicaciones por planta de $2.27 \mathrm{~kg}$ de pulpa de café $\left(9080 \mathrm{~kg} \mathrm{ha}^{-1}\right)$ e igual cantidad de gallinaza. El tratamiento orgánico extensivo (OE) involucra $2.27 \mathrm{~kg}$ de pulpa de café bajo las mismas especies arbóreas y un tercer tratamiento a plena exposición solar denominado convencional extensivo (CE) con aplicaciones por planta de 17 gramos $\left(68 \mathrm{~kg} \mathrm{ha}^{-1}\right)$ de fertilizante 27 9-18 en junio, 35 gramos (140 $\left.\mathrm{kg} \mathrm{ha}^{-1}\right)$ de 12-30-10 en septiembre y 20 gramos $\left(80 \mathrm{~kg} \mathrm{ha}^{-1}\right)$ de urea más 5 gramos $\left(20 \mathrm{~kg} \mathrm{ha}^{-1}\right)$ de $\mathrm{KCl}$ en octubre. Se utilizó un arreglo unifactorial en diseño de bloques completo al azar, evaluándose las variables materia orgánica, nitrógeno total, capacidad de intercambio catiónico, porosidad, retención de humedad, y diversidad de la macrofauna del suelo. Se efectuaron un muestreo de suelo en el mes de julio de 2009 y otro en enero de 2010. Los resultados fueron analizados por diferencias mínimas significativas y en el caso de la macrofauna a través del índice de Shannon-Wiener. Los programas estadísticos utilizados fueron InfoStat profesional y Past versión 1.29, éste último para el análisis de la biodiversidad del suelo. No se encontraron diferencias estadísticas en los componentes de la fertilidad física y química del suelo, pero se observa una tendencia a disminuir en el sistema manejado convencionalmente. La mayor diversidad de macrofauna se presentó en los sistemas manejados orgánicamente. El rendimiento de café oro se comportó de forma variada, siendo
ABSTRACT

The aim of the study was to compare the influence of three different coffee systems management on physical, chemical and biological soil fertility in Masatepe, Masaya, Nicaragua from July 2009 to January 2010. Treatment one (intensive organic) were two legume shadow species, Inga lauringa (Sw.) Willd (guaba) and Samanea saman (Jacq.) Merrill (genizaro), plus 2.27 kilograms (9 $080 \mathrm{~kg}$ $\mathrm{ha}^{-1}$ ) coffee pulp per plant plus same amount of chicken manure. Treatment two (extensive organic was 2.27 kilograms coffee pulp per plant and same shadow species and the third treatment (extensive conventional) was full sun exposure with 17 grams per plants (68 $\left.\mathrm{kg} \mathrm{ha}^{-1}\right)$ of fertilizer 27-9-18 in June, 35 grams (140 $\left.\mathrm{kg} \mathrm{ha}^{-1}\right)$ of fertilizer 12-30-10 in September and 20 grams $\left(80 \mathrm{~kg} \mathrm{ha}^{-1}\right)$ of urea +5 grams $\left(20 \mathrm{~kg} \mathrm{ha}^{-1}\right)$ of $\mathrm{KCl}$ In October per plant. A unifactorial arrangement with a completely random blocks design was used. Variables were organic matter, total nitrogen, cation exchange capacity, porosity, and water retention and macro fauna diversity. Soil samples were taken in July 2009 and January 2010, result was analyzed for least significant difference (LSD) and data from macro fauna were analyzed trough the Shannon-Wiener index. Statistical packages used were Professional InfoStat and Past version 1.29, the last one for soil biodiversity analysis. There were no significant differences for physical and chemical soil fertility but a tendency to reduction was observed in conventionally managed systems. The biggest diversity of macrofauna was observed in organically managed systems. Coffee green bean yield was variable, extensive conventional system was statistically higher in the first agricultural cycle with a yield of $305 \mathrm{~kg} \mathrm{ha}^{-1}$. There were no significant differences in the rest of periods, but there was a similar pattern on intensive organic systems compared with extensive conventional system. 
mejor estadísticamente el sistema convencional extensivo en el primer ciclo agrícola (2002-2003), con un rendimiento de 305 $\mathrm{kg} \mathrm{ha}^{-1}$. Posteriormente en el resto de los ciclos no se presentan diferencias significativas pero se observa un comportamiento alterno de mayor rendimiento entre el sistema orgánico intensivo y el convencional extensivo.

Palabras clave: Fertilidad física, química y biológica, sistemas agroforestal, suelo, sostenibilidad.

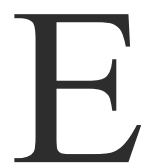
1 café (Coffea arabica L.), es uno de los cultivos de mayor importancia en Nicaragua. Genera importantes recursos económicos a través de sus exportaciones y contribuye con alrededor de 280 000 empleos directos e indirectos (ORGANICOOP, 2007).

En el ciclo agrícola 2010/2011 se produjeron 1,94 millones de quintales de café oro, en una superficie de 163 040 manzanas (114 574.84 hectáreas) con rendimientos

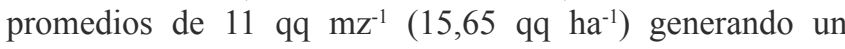
total de 438 millones de dólares, lo que representó un extraordinario incremento del $65.9 \%$ con respecto al ciclo anterior (CETREX, 2011).

La intensificación de la producción de este cultivo ha traído consecuencias negativas porque en muchas zonas se trabaja como monocultivos intensivos, donde los suelos están totalmente expuestos a los factores erosivos del ambiente. Además, las grandes cantidades de agroquímicos aplicados en estos sistemas, contribuyen a la contaminación de las aguas, disminución de la diversidad biológica del suelo (Muschler, 1999) y favorecen la aparición de plagas secundarias y cepas resistentes a pesticidas (Guharay et al., 1999).

Los altos rendimientos logrados en décadas anteriores no han sido sostenidos en el tiempo debido a la pérdida en la fertilidad de los suelos y al aumento en los precios de los insumos (ICAFE, 2004). Sin embargo, esto mejoraría si se proyectan sistemas de producción de café bajo manejo sostenible, como aquellos manejados orgánicamente y/o agroforestalmente.

Estos sistemas se caracterizan por tener árboles de usos múltiples asociados al cultivo, y su objetivo es disminuir la dependencia de insumos externos, reduciendo los costos de producción y favoreciendo la conservación de los recursos naturales de la finca; tales como el suelo, agua y la biodiversidad (Altieri, 1999).

Otro aspecto importante de estos sistemas es el mantenimiento de la fertilidad del suelo a través del reciclaje de nutrientes y mantenimiento de la materia orgánica, por medio de la producción y descomposición de la hojarasca y de los residuos de podas (Bolaños, 2001; citado por Gómez y Guerrero, 2007), lo que permite el mantenimiento o el aumento de la macrofauna en el suelo (Porras, 2006).

Estos sistemas se caracterizan por la asociación de varias especies en un mismo sitio, en diferentes grados de integración espacial y temporal (Imbach et al., 1989), las cuales son especies empleadas como soporte vivo, como
Keywords: Physical fertility, chemical and biological, agro forestry system, soil, sustainability.

sombra, para reducir el desgaste de los suelos y mantener un continuo suministro de nutrientes para el cultivo.

Lo anterior expuesto, permite suponer que el manejo de la fertilidad del suelo y del cultivo en arreglo agroforestal, es una estrategia imprescindible para orientar a los sistemas cafetaleros de una forma sostenible.

Esta investigación se hizo con el fin de determinar si cada sistema de manejo de café ejerce influencia en la fertilidad física, química y biológica del suelo y en el rendimiento del cultivo de café.

\section{MATERIALES Y MÉTODOS}

Descripción del área de estudio. Las áreas experimentales estaban ubicadas entre las coordenadas $11^{\circ} 54^{\prime \prime}$ de latitud norte y $86^{\circ} 09^{\prime}$ de longitud oeste, a una altitud de 455 metros sobre el nivel del mar. La zona está clasificada como baja y seca con suelos fértiles y precipitación promedio de 1400 $\mathrm{mm}$, temperatura anual promedio de $24^{\circ} \mathrm{C}$, con una estación seca de seis meses (Haggar y Staver, 2001).

Este estudio se inició en el año 2000 con el establecimiento de dos parcelas experimentales denominadas Níspero y Mamón, ubicadas en el Centro de Capacitación y Servicios Regional del Pacífico de Nicaragua conocido como Jardín Botánico y perteneciente en ese momento a UNICAFE. Una tercera parcela se estableció al siguiente año (2001), en el Centro Experimental Campos Azules (CECA) del INTA.

Para el presente trabajo fueron seleccionados los tratamientos en función del sistema de manejo, el tratamiento orgánico intensivo estuvo conformado por las especies de sombra Inga laurina y Samanea saman, más aplicaciones de $2.27 \mathrm{~kg}$ (9 $080 \mathrm{~kg} \mathrm{ha}^{-1}$ ) de pulpa de café por planta en la época seca y $2.27 \mathrm{~kg}$ de gallinaza por planta en el periodo de canícula (15 de julio-15 de agosto).

El tratamiento orgánico extensivo recibió a $2.27 \mathrm{~kg}$ de pulpa de café por planta aplicados en la época seca y bajo las mismas especies de sombra. El tratamiento convencional extensivo es café a plena exposición solar con aplicaciones en junio de $68 \mathrm{~kg} \mathrm{ha}^{-1}$ de fertilizantes mineral 27-09-18, en septiembre $140 \mathrm{~kg} \mathrm{ha}^{-1} 12-30-10 \mathrm{y}$ en octubre $80 \mathrm{~kg} \mathrm{ha}^{-1} \mathrm{de}$ urea más $20 \mathrm{~kg} \mathrm{ha}^{-1}$ de KCI.

La variedad de café establecida fue Pacas, la cual se estableció a una distancia de siembra a 2 metros entre surco y 1.25 metros entre planta, lo que determinó una densidad poblacional de 4000 plantas por hectárea, equivalente a 2810 plantas $\mathrm{mz}^{-1}$ 
Las especies de sombra fueron establecidas a 4 metros entre surco y 3.75 metros entre planta, para una densidad poblacional inicial de 666 plantas por hectárea.

Descripción del experimento. Se utilizó un Diseño de Bloques Completos al Azar (BCA) en arreglo unifactorial con tres repeticiones.

Variables evaluadas. En cada parcela se evaluó la fertilidad física, química y biológica del suelo así como el rendimiento del café. Para evaluar la fertilidad física se consideró la porosidad (\%) del suelo mediante cálculos matemáticos (Birecki y Col, 1968) $(\mathrm{n}=1-(\mathrm{Da} / \mathrm{Dr}) * 100)$ y la retención de humedad (CC) mediante el método de la ollas de presión. Para la fertilidad química del suelo la materia orgánica (\%) se determinó utilizando la metodología del método calorimétrico (Walkey y Black, 1947), nitrógeno total (\%) por la metodología de Kjeldhal y capacidad de intercambio catiónico (CIC) $\left(\mathrm{cmol}(+) \mathrm{kg}^{-1}\right)$ a través de solución extractora de acetato de amonio. La diversidad de macrofauna (fertilidad biológica) se estimó mediante la exploración del suelo, conteo e identificación en laboratorio y el rendimiento del café expresado en $\mathrm{kg} \mathrm{ha}^{-1}$ de café oro, considerando los últimos nueve ciclos agrícolas a partir del establecimiento del ensayo

Análisis estadístico. Las variables físicas, químicas y el rendimiento fueron sometidas a un análisis de varianza con separación de medias por diferencias mínimas significativas (DMS), con un margen de error del $5 \%(\mathrm{P} \leq 0.05)$ mediante el uso del programa estadístico InfoStat profesional. Para evaluar la diversidad biológica de la macrofauna del suelo, se utilizó el índice de Shannon-Wiener por medio del programa estadístico Past versión 1.29.

Formula Shannon-Wiener:

$$
H=-\sum_{i=1}^{S} i \cdot \log _{2}(p i)
$$

$$
p i=\frac{n i}{N}
$$

Dónde:

$n i=$ número de individuos en el sistema de la especie determinada i

$N=$ número total de individuos

$\mathrm{S}=$ número total de especies

\section{RESULTADOS Y DISCUSIÓN}

Materia orgánica del suelo (\%). No se establecieron diferencias estadísticas $(\mathrm{DMS}=4.25$ y 3.23$)$, pero sí una tendencia de disminución de los niveles de materia orgánica en el sistema convencional extensivo.

Los valores registrados oscilan entre 7.37 y $9.35 \%$, son clasificados según Fassbender (1987) como altos, quien establece ésta categoría cuando los porcentajes de materia orgánica del suelo sobrepasan el 5\% del volumen total del mismo.

Estos resultados concuerdan con los reportados por Centeno y Herrera (2005), quienes encontraron valores de materia orgánica entre 8 y $9 \%$ en suelos manejados bajo sistema orgánicos y con distintas especies de sombras; igualmente Paz y Sánchez (2007), registraron valores similares en suelos de origen volcánico y bajo diferentes tipos de sombras.

Estos niveles altos de materia orgánica es una condición particular de los suelos Andisoles (Cardona y Sadeghian, 2005), (Arnalds y Stahr, 2004).

La tendencia de disminuir en el sistema convencional extensivo se debe a la menor cantidad de materia orgánica que se incorpora en éste sistema, contrario a lo que ocurre en los sistemas orgánicos intensivo y extensivo, que aportan materia orgánica a través de la hojarasca de los árboles de sombra y del cafeto, más las enmiendas al suelo en forma de pulpa de café y gallinaza (figura 1).

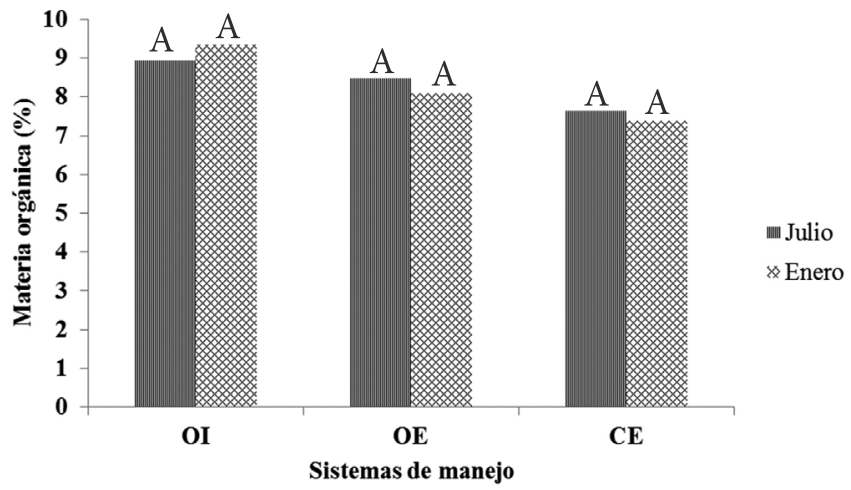

Figura 1. Materia orgánica del suelo por sistemas de manejo, julio 2009 y enero 2010.

Este comportamiento es reportado por Núñez (1985); Cairo y Quintero (1980), Gliessman (2002), quienes mencionan que los altos porcentajes de materia orgánica en los suelos se relacionan con la cantidad añadida de residuos frescos aportada por los árboles de sombra, siendo el aporte principal el que corresponde a la parte aérea, en este sentido los árboles leguminosos se caracterizan por su gran aporte de biomasa vegetal en forma de hojarasca (Henin, 1972). 
Nitrógeno total (\%). El análisis estadístico no indica diferencias significativas entre los tratamientos (DMS julio 0.17 y DMS enero 0.15) sin embargo, la tendencia es de que los contenidos de nitrógeno total del suelo disminuyan en el sistema convencional extensivo, registrándose el nivel más alto en el orgánico intensivo $(0.53 \%)$ y $0.36 \%$ en convencional extensivo.

Porras (2006), encontró la misma tendencia, siendo el mayor valor registrado de $0.57 \%$ en un sistema agroforestales con café orgánico y de $0.29 \%$ bajo un sistema convencional; igualmente George (2006), reportó valores similares en sistemas manejados orgánica y convencionalmente.

Según los criterios de clasificación de LABSA-UNA (2011), estos valores son considerados como altos (mayores de $0.15 \%$ ), y se ubican según Fassbender (1984), dentro del rango de concentraciones para suelos tropicales $(0.02 \%$ $2 \%)$.

Igual que en la variable materia orgánica del suelo, existe una tendencia de aumento en los contenidos de nitrógeno total en aquellos sistemas en los cuales existe un mayor aporte de biomasa; hecho que ocurre en los dos sistemas manejados orgánicamente. Esto es debido a los aportes que reciben como parte de las enmiendas, hojas, raíces y tallos provenientes de los árboles de sombra y los cafetos y se constituyen en la mayor parte de los materiales orgánicos aportados al suelo.

Ortega (1987), plantea que la variabilidad del nitrógeno en el suelo depende de la aportación de materia orgánica, y según Hernández y Rayo (2007), los sistemas cafetaleros bajo sombra de especies Inga laurina y Samanea saman manejados con insumos orgánicos, incrementan los niveles de nitrógeno con respecto a los sistemas a plena exposición solar.

Capacidad de intercambio catiónico [ $\left.\mathbf{c m o l}(+) \mathbf{~ k g}^{-1}\right]$. Según los análisis realizados en LABSA-UNA (2011), estos suelos presentan alta capacidad de intercambio catiónico, oscilando en el rango de 35 a $43 \mathrm{cmol}^{(+)} \mathrm{kg}^{-1}$ de suelo. De acuerdo con el análisis de los datos, no se establecen diferencias estadísticas por influencia de los sistemas de manejo.

Estos resultados fueron similares a los reportados por Centeno y Herrera 2005, quienes obtuvieron valores entre 34 y $46 \mathrm{meq} / 100$ gramos de suelo [ $\left.\left.\mathrm{cmol}(+) \mathrm{kg}^{-1}\right)\right]$ evidenciando la influencia del cultivo asociado y el efecto que tienen los residuos que estos generan sobre la fertilidad del suelo.

Kass (1998), expresa que los sistemas manejados bajo sombra son una fuente de materia orgánica que permite un incremento de la CIC en relación a otros sistemas de manejo. Esta conducta es debido a que la materia orgánica posee cargas negativas que confieren la capacidad de retención de grandes cantidades de cationes (Labrador, 1996).

Si la capacidad de intercambio catiónico se refiere a la capacidad que tiene un suelo de intercambiar sus cationes desde la solución del suelo al complejo de intercambio y viceversa (Fuentes, 1999) y considerando el aporte de elemento base producto de la mineralización de la materia orgánica, la CIC es considerada como normal bajo estas condiciones químicas de suelo.

La alta CIC que presenta este suelo, es justificada por Bertsch (1987), quien señala que los valores de CIC en los suelos fluctúan en el rangos de $5-50 \mathrm{cmol}(+) \mathrm{kg}^{-1}$ de suelo, siendo 50 el valor más alto, generalmente presente en suelos con gran contenidos de arcillas y alta capacidad de retención de agua, condición que según Kass (1998), es normal en suelos Andisoles por la presencia de arcillas alófana que confiere alta capacidad de intercambio de cationes.

Porosidad del suelo (\%). Los valores de porosidad registrados son clasificados por Cairo (1995) como muy altos, ya que sobrepasan el $65 \%$ del espacio vacío; sin embargo, no se establecen efectos significativos de los sistemas de manejo sobre ésta propiedad.

Los valores de porosidad tienen una tendencia de ser mayores en los sistemas manejados orgánicamente, debido a que la materia orgánica y la actividad biológica, (macro y micro) tienen una relación directa con esta propiedad (Labrador, 1996). Estos resultados concuerdan con los reportados por Cardona y Sadeghian (2005), quienes encontraron mayores valores de porosidad (65 y $67 \%$ ) en suelos manejados orgánicamente, demostrando que al aumentar el contenido de materia orgánica, el volumen del suelo es mayor debido a un aumento en el número de poros.

La porosidad es determinante para que se manifiesten favorablemente condiciones físicas, químicas y biológicas del suelo, que permiten un buen crecimiento y desarrollo de los cultivos, así lo explican Cairo y Quintero (1980), Painedy Wild (1992), quienes señalan que suelos con altos valores de porosidad, favorecen la aireación, conservación de la humedad, el intercambio entre el cultivo y el suelo, así como el crecimiento de raíces y el buen desarrollo de la vida edáfica.

Retención de humedad (\%). La tendencia en la retención de humedad es a la disminución en el sistema manejado convencionalmente. De los tres sistemas, aquellos que son manejados orgánicamente presentaron un ligero incremento en la capacidad de retención, aunque sin diferencias estadísticas, comportamiento atribuido a los contenidos de materia orgánica y a la sombra de los árboles.

Este comportamiento es respaldado por Guharay et al., (2000), quienes indican que la sombra de los árboles y la hojarasca protegen los suelos de altas temperaturas y cambios bruscos en la humedad del suelo.

Los registros oscilaron entre 39 y $47 \%$, valores considerados por Cairo (1995), como de mediana capacidad para el convencional extensivo y de alta capacidad de retención de agua para los sistemas orgánicos. 
Esto se atribuye al contenido de materia orgánica existente en estos suelos y a los residuos vegetales aportados por las especies de sombra en los sistemas orgánicos, que permiten regular la temperatura, disminuir las pérdidas de agua por evaporación y aumentar la porosidad del suelo.

Paz y Sánchez (2007) encontraron valores mayores de retención de humedad en suelos manejados orgánicamente y con sombra. Coincidentemente estos resultados son reportados por Cardona y Sadeghian (2005), Hernández y Rayo (2007), quienes encontraron que suelos con altos contenidos de materia orgánica permiten una mayor capacidad de retención de agua, demostrando con esto que la cantidad de agua almacenada en los suelos, es condicionada por los contenidos de materia orgánica.

Los suelos originados a partir de ceniza volcánica dan origen a suelos con alta fertilidad química y gran retención hídrica (Labrador, 1996), lo que se debe a la alta acumulación de materia orgánica en su superficie, debido a que las alófanas presentes adsorben fuertemente las sustancias orgánicas, limitando su degradación (Unigarro, 2005). La acumulación de hojarasca favorece positivamente la retención de humedad. Quiroga et al., (2005), señalan que la retención de humedad depende principalmente del tipo de suelo, del sistema de manejo y el régimen pluviométrico.

Cardona y Sadeghian (2005), indican que los suelos que albergan cafetales con sombra poseen mejores condiciones de retención de humedad, porosidad, estabilidad y permeabilidad que aquellos suelos con cultivos a libre exposición solar.

Macrofauna del suelo. La tabla 1, muestra los índices de Shannon-Wiener por tratamientos y épocas de muestreo, y se observa que la diversidad de la macrofauna del suelo es mayor en los sistemas que reciben abonos orgánicos y que son manejados agroforestalmente.

Esto coincide con lo reportado por George (2006), quien encontró mayor número de especies, géneros y familias en fincas manejadas orgánicamente, en comparación con las fincas bajo manejo convencional. En un estudio realizado en Cuba Cabrera et al., 2011, registró mayor riqueza y abundancia de macroorganismos en suelos con abundante cobertura que en suelos con poca cobertura.

Estos mismos resultados fueron encontrados en sistemas agroforestales con café por Sánchez et al., (2005), citado por Porras (2006) en un estudio realizado en Costa Rica, quienes encontraron mayores individuos en parcelas orgánicas comparado con sistemas convencionales. Al registrar menor cantidad de individuos Sánchez et al., (2005) concluyeron que las áreas con mayor cobertura de especies vegetales manifiestan mayor diversidad en la macrofauna del suelo.
Tabla 1. Efecto de tres sistemas de manejo sobre la macrofauna del suelo por época y sistema de manejo según el índice de ShannonWiener

\begin{tabular}{lcccc}
\hline Tratamientos & \multicolumn{5}{c}{ Época de muestreo } \\
\hline & Mayo & Julio & Octubre & Enero \\
\hline Orgánico intensivo & 1.01 & 1.84 & 1.94 & 1.77 \\
Orgánico extensivo & 1.06 & 2.02 & 1.80 & 1.33 \\
Convencional extensivo & 1.00 & 1.76 & 1.75 & 1.17
\end{tabular}

Las poblaciones de la macrofauna en los suelos varían en el espacio y en el tiempo, pudiendo cambiar según sea la disponibilidad de nutrientes, condiciones del ambiente y las especies vegetales involucradas (USDA, 1999).

Además los niveles superiores en los componentes de la fertilidad física y química, principalmente de materia orgánica, permiten este comportamiento. Así lo reporta Abbott y Murphy (2007), indicando que la materia orgánica permite incrementar o disminuir la fauna del suelo en función de las cantidades y la calidad de la misma.

La fauna edáfica forma parte de las cadenas y redes tróficas que varían en complejidad de acuerdo con las condiciones bióticas y abióticas del suelo como el tamaño de agregados de las partículas, la humedad del suelo, concentraciones de oxígeno, variaciones climáticas (Jiménez et al., 2003), estado de desarrollo, grado de alteración del ecosistema y contenidos de materia orgánica (Labrador, 1996).

La mayoría de los organismos del suelo pertenece a la cadena de los detritívoros involucrados en la descomposición de la materia orgánica, desempeñando un importante papel en la fertilidad y estabilidad del suelo; otros grupos son depredadores, omnívoros fitófagos, rizófagos, geófagos, y su principal función es mantener el equilibrio de las poblaciones y del medio edáfico en general (Coleman, 2004).

Las plantas y los desechos orgánicos proveen los hábitats y los alimentos para la macrofauna, y el adecuado contenido de materia orgánica favorece no solo las propiedades físicoquímicas, sino también proporciona una fuente energética que estimula la actividad de los invertebrados del suelo.

La cobertura arbórea en el ordenamiento de los ecosistemas, es de suma importancia, pues garantiza la entrada continua y abundante de material orgánico vegetal y un microambiente más favorable para el establecimiento de la macrofauna del suelo (Brown, 2001).

Rendimiento de café oro $\left(\mathrm{kg} \mathrm{ha}^{-1}\right)$. La figura 2 muestra el comportamiento de la producción en el tiempo y se presenta de manera similar en los tres sistemas, con aumentos y disminuciones en los ciclos agrícolas. 
El análisis de varianza muestra que solo en el primer ciclo agrícola existen diferencias significativas, $(\mathrm{p}=0.047$, $\mathrm{DMS}=200$ ). La mejor categoría la presenta el sistema convencional extensivo con un rendimiento de $305 \mathrm{~kg} \mathrm{ha}^{-1}$, seguido en una misma categoría estadística por el sistema orgánico intensivo con $79 \mathrm{~kg} \mathrm{ha}^{-1} \mathrm{y}$ el orgánico extensivo con $70 \mathrm{~kg} \mathrm{ha}^{-1}$ de café oro.
El comportamiento en el primer ciclo se podría atribuir a la disponibilidad de nutrientes de los portadores sintéticos, los cuales pueden ser absorbidos por las plantas de café de forma inmediata cuando estos encuentran condiciones de humedad adecuada en el suelo, como lo reporta Fischersworring y RoBKamp (2001).

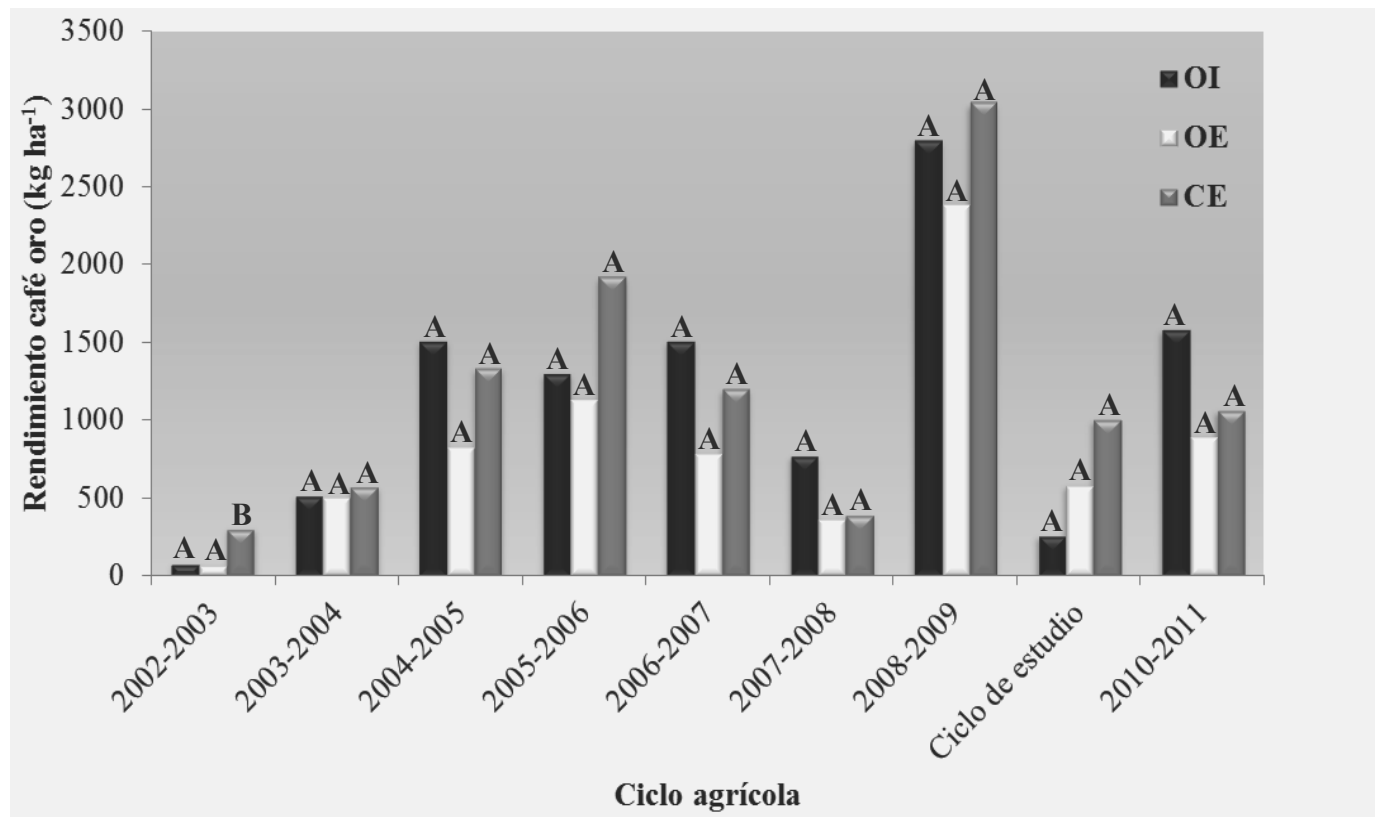

Figura 2. Rendimiento de café oro por ciclo agrícola y sistema de manejo, Masatepe, Masaya 2002-2011.

Durante este ciclo agrícola la cantidad de nutrientes provenientes de la hojarasca de los árboles y las enmiendas al suelo, no proporcionan la cantidad de elementos esenciales suficientes como para igualar los rendimientos del sistema convencional.

El aumento continuo en los primeros ciclos agrícolas se puede atribuir a que las plantas están en pleno crecimiento y las cosechas aumentan en la medida que avanza la edad del cultivo. Para el caso del descenso de los rendimientos, esto es atribuido al comportamiento de las condiciones ambientales cambiantes y fisiológicas del café, principalmente a las precipitaciones y al comportamiento bienal del cultivo.

Los rendimientos en los ciclos agrícolas 2003-2004, 2005-2006, 2008-2009 y 2009-2010 fueron mayores en el sistema convencional extensivo.

Muschler (1999), indica que los cafetales a plena exposición solar tienen mayor producción y rápido crecimiento siempre y cuando tengan buenas condiciones de altitud y temperaturas.

Estas diferencias en la productividad del café entre los sistemas de manejo fueron reportadas por Cardoza y Jiménez (2007), donde la producción convencional fue mayor que en los sistemas orgánicos, igual lo reporta Merlo (2007), en un estudio realizado en Costa Rica registrando mayores rendimientos en fincas manejadas convencionalmente en comparación a fincas con manejo orgánico.
Al analizar los ciclos agrícolas 2004-2005, 2006-2007, 2007-2008, 2010-2011 los rendimientos fueron mayores en el sistema orgánico intensivo, ubicándose en una posición intermedia el sistema orgánico extensivo.

Este comportamiento se debe a las precipitaciones ocurridas y su distribución en cada ciclo agrícola, las cuales condicionan la floración oportuna de las plantas y su fructificación, además propician la mineralización de la materia orgánica y disponibilidad de nutrientes; todo esto sumado a una mayor contribución de biomasa en forma de hojarasca y del manejo de la poda de las especies de sombra y su aporte como fijadores de nitrógeno.

Los agroecosistemas de café con diversidad vegetal, son por lo general, menos productivos pero ciertamente más estables y sostenibles que los monocultivos, por el rol benéfico de los árboles de sombra para conservar la materia orgánica, pues ésta influye sustancialmente en la fertilidad biológica, química y física del suelo; de allí la importancia de implementar prácticas que favorezcan su conservación y que incrementen la eficiencia de los procesos como la fijación biológica de nitrógeno y la simbiosis micorrítica para reducir el uso de fertilizantes y otros agroquímicos (Vaast y Snoeck 1999; citado por Merlo, 2007), (Muschler, 1999), (Fischersworring y RoBkamp 2001). 
Rendimiento acumulado de café oro $\left(\mathrm{kg} \mathrm{ha}^{-1}\right)$. El sistema convencional extensivo presentó el mayor rendimiento acumulado (10 $824 \mathrm{~kg} \mathrm{ha}^{-1}$ ) seguido por el sistema orgánico intensivo (10 $\left.347 \mathrm{~kg} \mathrm{ha}^{-1}\right)$ y luego el orgánico extensivo con 7 $577 \mathrm{~kg} \mathrm{ha}^{-1}$. Según Fassbender (1987), en términos generales los rendimientos de los sistemas orgánicos podrían ser entre 10 y $20 \%$ menores que los de un sistema convencional, sin embargo, nuestro análisis refleja que la diferencia entre el sistema manejado convencionalmente y el orgánico intensivo es de $495 \mathrm{~kg}$ oro ha ${ }^{-1}$ equivalente a $4.57 \%$. Esta mínima diferencia generalmente es aceptada porque la producción es más sostenible y con menos efectos negativos sobre el ambiente (Gliessman, 2002).

Este margen mínimo de diferencia nos permite afirmar que los sistemas manejados con sombra e insumos orgánicos nos permiten proyectar nuestros rendimientos de forma sostenible, lo que se traduce en la conservación de los recursos naturales, aumento de la diversidad y mejora en los ingresos económicos al reducir los costos de fertilizantes sintéticos y la comercialización y uso de los subproductos del sistema como son combustible (leña), postes, estacas y madera.

\section{CONCLUSIONES}

No se registran cambios significativos en los componentes de la fertilidad física (porosidad y retención de humedad) y química (materia orgánica, nitrógeno total y capacidad de intercambio catiónico), pero si una tendencia en el sistema convencional extensivo a manifestar una menor fertilidad del suelo.

Se registró mayor diversidad de macrofauna en los sistemas manejados de forma orgánica por efecto de los mayores niveles de materia orgánica del suelo.
El rendimiento fue estadísticamente diferente únicamente en el primer ciclo agrícola destacándose el sistema convencional extensivo con $305 \mathrm{~kg} \mathrm{ha}^{-1}$ de café oro.

Se registró un comportamiento alterno de mayor rendimiento entre el sistema orgánico intensivo y el convencional extensivo.

El rendimiento acumulado en el sistema orgánico intensivo en los nueve ciclos agrícola fue superado escasamente en un $4.57 \%$ por el sistema convencional extensivo, lo que demuestra que este tipo de manejo permiten proyectar los rendimientos de manera sostenible.

\section{RECOMENDACIONES}

Establecer sistemas cafetaleros con enfoque orgánico intensivo y con especies de árboles fijadores de nitrógeno.

Estudiar el efecto de la aplicación de los abonos orgánicos y sintéticos sobre otros componentes de la fertilidad física (densidad aparente, densidad real, infiltración de agua), químicas $(\mathrm{C} / \mathrm{N}, \mathrm{pH}$, Carbono orgánico, $\mathrm{P}, \mathrm{K}, \mathrm{Ca}$, $\mathrm{Mg}, \mathrm{S}$ y micronutrientes) y biológica (microorganismos), para conocer su comportamiento y el efecto sobre el suelo y el cultivo.

\section{AGRADECIMIENTO}

Agradecemos a la Universidad Nacional Agraria (UNA), al pueblo y gobierno de Suecia y a la Agencia Sueca de Cooperación Internacional para el Desarrollo (Asdi), por el financiamiento otorgado para la realización de este trabajo de investigación.

\section{REFERENCIAS BIBLIOGRÁFICAS}

Abbott, LK; Murphy, DV. 2007. Soil biological fertility a key to sustainable land use in agricultura. Ed. Springer. Dordrecht. The Netherlands. $264 \mathrm{p}$.

Altieri, MA. 1999. Agroecology the science of sustainable agriculture. westview Process, Boulder. 257 p.

Arnalds, O; Stahr, K. 2004. Volcanic soil resources: occurrence, development, and properties. Catena, CO. 56 p.

Bertsch, F. 1987. Manual para interpretar la fertilidad delos suelos. San José, CR. 120 p.

Brown, G. 2001. Diversidad y rol funcional de la macrofauna edáfica en los ecosistemas tropicales mexicanos. Acta Zoológica Mexicana. MX.

Cabrera, G; Robaina, N; D. Ponce de León. 2011. Riqueza y abundancia de la macrofauna edáfica en cuatro usos de la tierra en las provincias de Artemisa y Mayabeque, Cuba. (en línea). Consultado 25 may. 2012. Disponible en http://scielo.sld.cu/scielo. php?pid=S0864-03942011000300007\&script=sci arttext

Cairo, P. 1995. La fertilidad física de suelo y la agricultura orgánica en el trópico. Universidad Nacional Agraria. Managua, NI. 228 p.

Cairo, PG; Quintero. 1980. Suelos. Ed. Pueblo y educación. 2 reimp. La Habana, CU. 367 p.

Cardona, AC; Sadeghian KH. 2005. Caracterización de la fertilidad del suelo en monocultivo de café (Coffea arabica L) y bajo sombrío de guamo (Inga sp). (en línea). Consultado 25 feb. 2012. Disponible enhttp://revistas.udistrital.edu.co/ojs/index.php/colfor/article/ view/3049/4602

Cardoza, OM; Jiménez, ME. 2007. Evaluación de rendimiento del grano de café (Coffea arabica L.) bajo la influencia de diferentes manejos agroforestales en Masatepe. Tesis Ing. Agr. Universidad Nacional Agraria, Facultad de Agronomía. Managua, NI. 61 p.

Centeno, RE; Herrera, H. 2005. Caracterización biofísica y suelos de los sistemas cafetaleros en la zona baja húmeda de Matagalpa. Tesis Ing. Agr. Universidad Nacional Agraria, Facultad de Agronomía. Managua, NI. 79 p. 
CETREX (Centro de Trámites de Exportaciones). 2011. Estadísticas de café exportaciones (en línea). Consultado 26 feb. 2012. Disponible en http://cetrex.gob.ni/website/servicios/cafe/cafe.jsp

Coleman, DC. 2004. Fundamentals of Ecology 2nd ed. USA. Elsevier, academic press. 386 p.

Fassbender, HW. 1987. Modelos edafológicos de sistema agroforestales. 2 ed. San José, CR. 484 p.

Fassbender, HW. 1984. Química de suelos. Con énfasis en suelos de América Latina.

Instituto interamericano de cooperación para la agricultura, 4 reimp. San José, CR. 398 p.

Fischersworring, H; RoBKamp, R. 2001. Guía para la caficultura ecológica. 1 ed. Alemania. 149 p.

Fuentes, JL. 1999. El suelo y los fertilizantes. Ministerio de agricultura, pesca y alimentación. 5 ed. Mundi-prensa. Madrid, ES. 352 p.

George, A. 2006. Estudio comparativo de indicadores de calidad de suelo en fincas de café orgánico y convencional en Turrialba, Costa

Rica. San José, CR. (en línea). Consultado 25 may. 2012. Disponible enhttp://201.207.189.75/repdoc/A0834e/A0834e.pdf

Gliessman, SR. 2002. Agroecología. Procesos ecológicos en agricultura sostenible. CATIE. Turrialba, San José, CR. 359 p.

Gómez, GR; Guerrero, GW. 2007. Efecto de diferentes niveles de insumos y tipos de sombra sobre el comportamiento de las principales plagas del cultivo del café, ciclo 2006-2007. Tesis Ing. Agr. Universidad Nacional Agraria, Facultad de Agronomía. Managua, NI. $62 \mathrm{p}$.

Guharay, F; Monterrey, J; Monterroso, D; Staver, C. 2000.Manejo integrado de plagas del cultivo del café. Managua, NI. 50 p.

Guharay, F; Monterroso, D; Staver, C. 1999. Designing pest-supressive multi-strata perennial crop systems. In: Multi-strata agroforestry systems with perennial crops. Proceedings; International symposium on Multi-strata agroforstry systems with perennial crops. CATIE, Turrialba, CR. 84 p.

Haggar, J; Staver, CH. 2001. Sostenibilidad y sinergismo en sistemas agroforestales con café. Estudio de interacciones entre plagas, fertilidad del suelo y árboles de sombra. Agroforestería en las Américas. 51 p.

Henin, S. 1972. El estado físico del suelo y sus consecuencias agronómicas. Mundi-prensa. Madrid, ES. 245 p.

Hernández, MR; Rayo, BA. 2007. Tasa de mineralización del nitrógeno en el suelo bajo diferentes manejos agroforestales con café (Coffea arabica L.) realizado 2002 y 2006 en el pacifico sur de Nicaragua. Tesis Ing. Agr. Universidad Nacional Agraria, Facultad de Agronomía. Managua, NI. 56 p.

ICAFE (Instituto del Café de Costa Rica). 2004. Informe sobre la actividad cafetalera de Costa Rica. San José, CR. 70 p.

Imbach, AC; Fassbender, HW; Borel, R; Beer, J; Bonnemann, A. 1989. Modelling agroforestry systems of cacao (Theobroma cacao L) with laurel (Cordia alliodora) and cacao with poro (Erythrinapo eppigiana) in Costa Rica. IV. Water balances, nutrient inputs and leaching. Agroforestry Systems. 267 p.

Jiménez, JJ; Decaens, T; Thomas, RJ; Lavelle, P. 2003. La macrofauna del suelo. Las comunidades de macroinvertebrados del suelo en las sabanas neotropicales de Colombia. Ed, Jiménez. Bogotá, CO. 444 p.

Kass, CL. 1998. Fertilidad de suelos. San José, CR. 277 p.

Labrador, MJ. 1996. La materia orgánica en los agrosistemas. Ministerio de agricultura, pesca y alimentación. Mundi-prensa. Madrid, ES. 174 p.

LABSA-UNA. (Laboratorio de Suelo y Agua), (Universidad Nacional Agraria). 2011. Análisis físico, químico y biológico del suelo. Managua, NI.

Merlo, CM. 2007. Comportamiento productivo del café (Coffea arabica L variedad caturra), el poró (Erythrinapo eppigiana) el amarillon (Terminalia amazónica) y el Cashá (Choroleuco neurycydum) en sistemas agroforetales bajo manejo convencional y organicos en Turrialba, Costa Rica. (en línea). Consultado 30 may. 2012. Disponible en http://orton.catie.ac.cr/repdoc/A1306e/A1306e.pdf

Muschler, RG. 1999. Sombra o sol para un café sostenible. Un enfoque de una vieja discusión. Boletín PROMECAFE. 81 p.

Núñez, S. 1985. Fundamentos de la edafología. San José, CR. 2ed. 185 p.

ORGANICOOP. 2007. Producción cafetalera sigue soportando una fuerte crisis. (en línea). Consultado 04 feb 2012. Disponible en http:// organicop.blogspot.com/2007/09/caf-de-nicaragua.html

Ortega, E. 1987. Química de suelos. Patenac AC. Chapingo, MX. 52 p.

Pained, D; Wild, A. 1992. Estructura del suelo, laboreo y comportamiento mecánico condiciones del suelo y desarrollo de las plantas, según Russell. Madrid, ES. Edición Mundi-prensa. 1045 p.

Paz, IE; Sánchez PM. 2007. Relación entre dos sistemas de sombrío de café y algunas propiedades físicas del suelo en la meseta de Popayán. (en línea). Consultado 24 feb. 2011. Disponible enhttp://web.ebscohost.com/ehost/pdf?vid=8\&hid=13\&sid=2a783d1b81eb-46f0-a 73b- 2c7e4b465b77\%40sessionmgr107

Porras, VC. 2006. Efecto de los sistemas agroforestales de café orgánico y convencional sobre las características de suelo en el corredor bilógico. Turrialba-Jiménez, Costa Rica. (en línea). Consultado 29 mar. 2012. Disponible en http://orton.catie.ac.cr/repdoc/A0831e/ A0831e.pdf

Quiroga, AR; Funaro, DA; Fernández, R; Noellemeyer, EJ. 2005. Factores edáficos y de manejo que condicionan la eficiencia del barbecho en la región pampeana. (en línea) consultado 02 jun. 2012. Disponible en http://www.scielo.org.ar/pdf/cds/v23n1/v23n1a09.pdf

Unigarro, A. 2005. Evaluación de la calidad de un suelo mediante la determinación de algunas propiedades biológicas, químicas y físicas, en el santuario de flora y fauna galeras, Narino. Universidad Nacional de Colombia. Bogotá, CO. 60 p.

USDA (United States Department of Agriculture). 1999. Guía para la evaluación de la calidad y salud del suelo. 82 p. 